\title{
Optical switching studies of an azobenzene rigidly linked to a hexa-peri-hexabenzocoronene derivative in solution and at a solid-liquid interface
}

\author{
Min Ai • Susie Groeper • Wei Zhuang • Xi Dou • \\ Xinliang Feng · Klaus Müllen · Jürgen P. Rabe
}

Received: 9 April 2008 / Accepted: 9 July 2008 / Published online: 2 September 2008

(C) The Author(s) 2008. This article is published with open access at Springerlink.com

\begin{abstract}
An azobenzene moiety rigidly linked to a hexa-peri-hexabenzocoronene (HBC) derivative has been switched optically between its trans- and cis-conformations in solution. Crystalline monolayers of the flat lying transconformer have been obtained at the interface between the basal plane of graphite and an organic solution. However, from the illuminated solution no cis-conformer was observed adsorbed to the interface, indicating that the resulting cis- conformer is not thermodynamically stable at this interface, possibly due to the competition with the coexisting trans-conformers. Therefore, two-dimensional honeycombs self-assembled from derivatives of three-fold symmetric polycyclic aromatic hydrocarbons were investigated, which may be employed as templates, providing the necessary space for conformational switching of an azobenzene moiety, and tune the current-voltage characteristics through the aromatic cores. As a first step towards this goal, the capability of the template to host single molecular guests was studied by filling the voids with individual coronene molecules.
\end{abstract}

PACS 81.07.Nb - 81.16.Dn · 68.08.-p · 68.37.Ef ·

42.79.Ta $\cdot 85.65 .+\mathrm{h} \cdot 37.70 .+\mathrm{i}$

\footnotetext{
M. Ai · S. Groeper · W. Zhuang · J.P. Rabe $(\varangle)$

Department of Physics, Humboldt University Berlin, Newtonstr.

15, 12489 Berlin, Germany

e-mail: rabe@physik.hu-berlin.de

X. Dou $\cdot$ X. Feng $\cdot$ K. Müllen

Max Planck Institute for Polymer Research, Ackermannweg 10, 55128 Mainz, Germany
}

\section{Introduction}

Current-voltage characteristics of hybrid molecular diodes, i.e., single molecules in nanogaps have been studied in nanoscopic metal-, break-, or STM junctions [1-8]. Recently, the current-voltage characteristics through a single hexa-peri-hexabenzocoronene (HBC) moiety have been controlled via the dipole moment of covalently linked charge transfer complexes at a solid-liquid interface [9]. In this case, the complex formation and consequently the switching were due to the diffusion of an electron donor in the solution towards the acceptor at the interface, which is a random and slow process. Light-induced switching of a dipole moment at the interface should allow for a better controlled switching. A suitable candidate is azobenzene, which can be switched optically and electrically from a trans- to a cisconformation [10-13], accompanied by a change in dipole moment.

We report here on the optical switching of an azobenzene moiety rigidly linked to an $\mathrm{HBC}$ derivative in solution, the immobilization of the flat lying trans-conformer in a crystalline monolayer at the interface between the basal plane of graphite and an organic solution, and its submolecularly resolved in-situ STM-imaging. Since the cis-conformer of the molecule did not adsorb in ordered monolayers, we selfassembled stable two-dimensional honeycombs from larger polycyclic aromatic hydrocarbons, which may serve as templates, providing the necessary space for conformational switching of an azobenzene moiety on the surface, and further allow tuning the current-voltage characteristics through the aromatic cores.

\section{Experimental}

Compounds 1 and 2 (Figs. 1 and 2) were synthesized according to procedures, which will be provided elsewhere; 
the synthesis of $\mathbf{3}$ has been described before [14]. Coronene has been obtained commercially (99\%, Aldrich). STM experiments at the interface between almost saturated solutions in 1, 2, 4-trichlorobenzene and the basal plane of highly oriented pyrolytic graphite (HOPG) were carried out under ambient conditions [7, 15] using a home-made STM employing commercial electronics (Omicron Vakuumphysik $\mathrm{GmbH}$, Taunusstein, Germany). The tips were prepared by mechanically cut Pt/Ir (80:20) wires. HOPG (grade ZYH, Advanced Ceramics, Cleveland, Ohio, U.S.A) was freshly peeled with adhesive tape prior to an experiment. STM images were obtained at negative sample bias with respective to the tip. The lattice of the underlying HOPG has been visualized during the measurements by simply changing the tunneling parameters, which allowed calibration of the piezo in the $x y$ plane in-situ. Unit cells were averaged over several images after their correction for the piezo drift (using SPIP Scanning Probe Image Processor, Version 1.911, Image Metrology ApS, Lyngby, Denmark).

The photoisomerization from trans- to cis-azobenzene within compound $\mathbf{1}$ was carried out in dilute dichloromethane solution $\left(1.02 \times 10^{-5} \mathrm{M}\right)$ by irradiating the solution in-situ at room temperature with a CAMAG UV lamp (8 W) light source emitting at $366 \mathrm{~nm}$. The UV spectra were recorded using a UV/Vis/NIR Perkin-Elmer Lambda 900 spectrometer. In almost saturated solutions of 1, 2, 4-trichlorobenzene, the photoisomerization was accomplished using a Shimadzu RF-5001 PC fluorescence spectrometer light source $450 \mathrm{~nm}$. The UV spectra were recorded with Shimadzu UV 2102 PC UV-Vis spectrometer.
Fig. 1 Chemical formulae of an azobenzene moiety rigidly linked to a

hexa-peri-hexabenzocoronene derivative, $\mathbf{1}$, which can be switched reversibly between its dichloromethane and

1, 2, 4-trichlorobenzene solution trans- and cis-conformations in
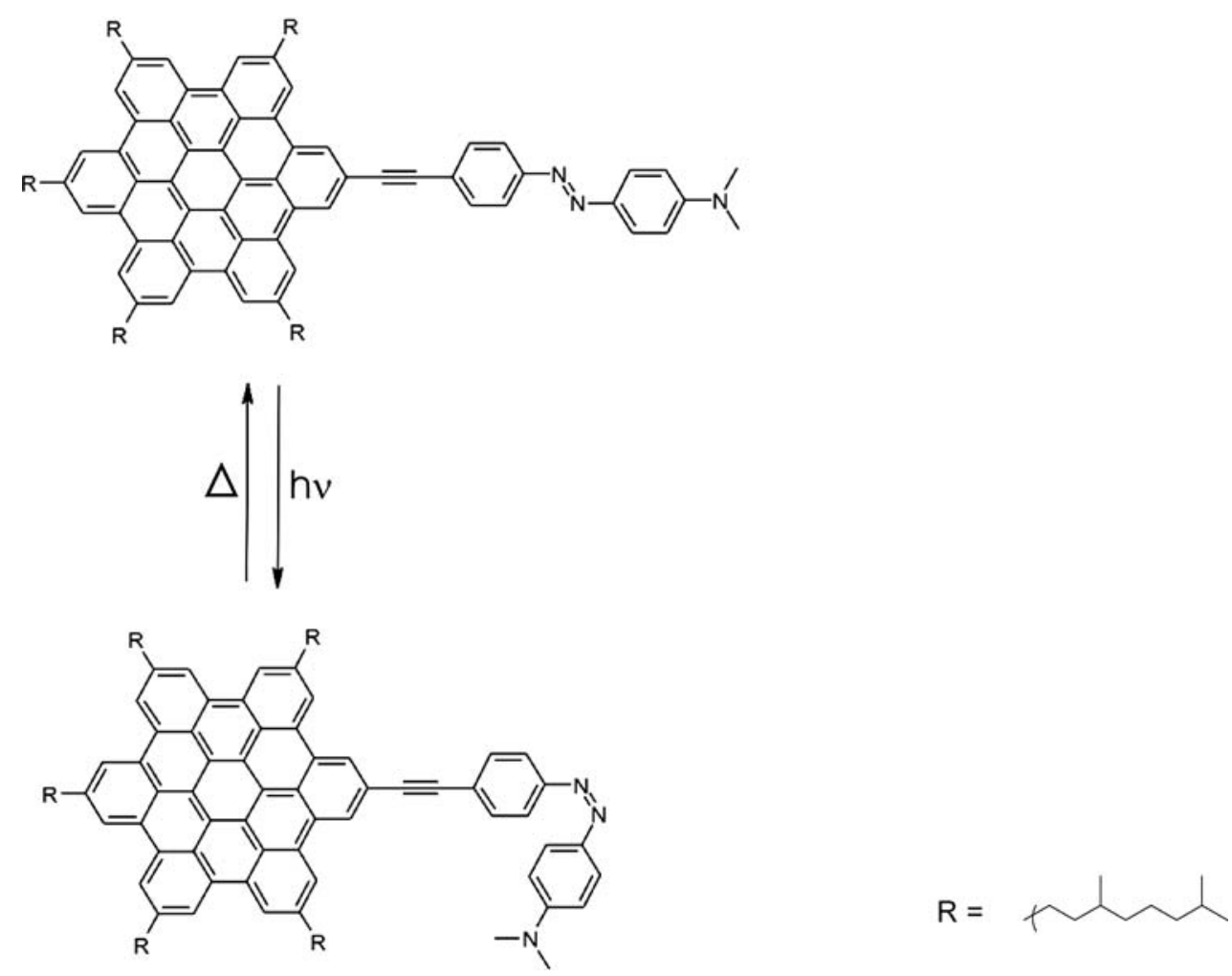

Fig. 2 Chemical formulae of $\mathbf{2}$, trizigzagHBC $(\mathrm{Ph} \text {-esterI })_{3}, \mathbf{3}$, trizigzagHBC $\left(\mathrm{Ph}-\mathrm{C}_{8,2}\right)_{6}$, and $\mathbf{4}$, coronene
2 trizigzagHBC(Ph-esterl) ${ }_{3}$ :<smiles>[R12]Cc1ccc(CCC(C)CCCC(C)C)cc1</smiles><smiles>c1cc2ccc3ccc4ccc5ccc6ccc1c1c2c3c4c5c61</smiles> 


\section{Optical switching in solution}

The azobenzene moiety rigidly linked to an HBC derivative, 1, has been optically switched between its trans- and cis-conformations in dilute dichloromethane solution with light of a wavelength of $366 \mathrm{~nm}$. Figure 3a displays the optical absorption spectra as functions of illumination time up to 5 hours and the thermal back reaction time of about 15 seconds at $60^{\circ} \mathrm{C}$. The strong peak around $366 \mathrm{~nm}$ [16] is attributed to the chromophore consisting of the $\mathrm{HBC}$ and the alkyne group, which does not change much upon illumination. A broader peak around $450 \mathrm{~nm}$ decreases substantially upon illumination, while on the same time a redshifted peak appears around $550 \mathrm{~nm}$, causing an isosbestic point slightly above $500 \mathrm{~nm}$. We assign the initial absorption around $450 \mathrm{~nm}$ to the $\pi-\pi^{*}$ transition of the transconformation of the amine-terminated azobenzene and the absorption appearing at $552 \mathrm{~nm}$ to the $\mathrm{n}-\pi^{*}$ transition of the photoinduced cis-conformation. Thermal isomerism from the photogenerated cis- to the trans-conformer occurs after heating at $60^{\circ} \mathrm{C}$ within seconds [10]. Obviously there is a strong electronic coupling between the $\mathrm{HBC}$, the alkyne and the azobenzene, since the excitation of the former switches the latter.

Since the STM experiments were carried out in a solution of 1,2, 4-trichlorobenzene, the photoreaction of compound 1 was also investigated within this solvent. Upon illumination at $366 \mathrm{~nm}$, no cis-azobenzene was formed, while irradiation at $450 \mathrm{~nm}\left(\pi-\pi^{*}\right.$ transition of the transconformer) bleached the trans-band and led to the formation of the band around $552 \mathrm{~nm}$, attributed to the cisconformer (Fig. 3b). Upon thermal treatment at $60^{\circ} \mathrm{C}$, the trans-conformer reappeared. Apparently, the switching depends on the solvent and possibly on the molecular aggregation.

In both cases, while the isosbestic points indicate a clean switching between the two conformers under these conditions, in poorer solvents like alkanes, no switching is observed. We attribute this to aggregate formation, which prevents the switching, probably due to steric hindrance [17].

\section{Monolayers at solid-liquid interfaces}

In order to be able to follow the optical switching on the molecular scale, we assembled compound $\mathbf{1}$ at the interface between a solution in 1,2,4-trichlorobenzene and the basal plane of HOPG. Figure 4 displays in-situ STM images with a double row pattern including grain boundaries, which reflect the threefold-symmetry of the substrate (Fig. 4a) and high resolution images revealing the nanophase separation between the flat lying HBC cores on the one hand and the azobenzene moieties with their rigid linker on the other hand
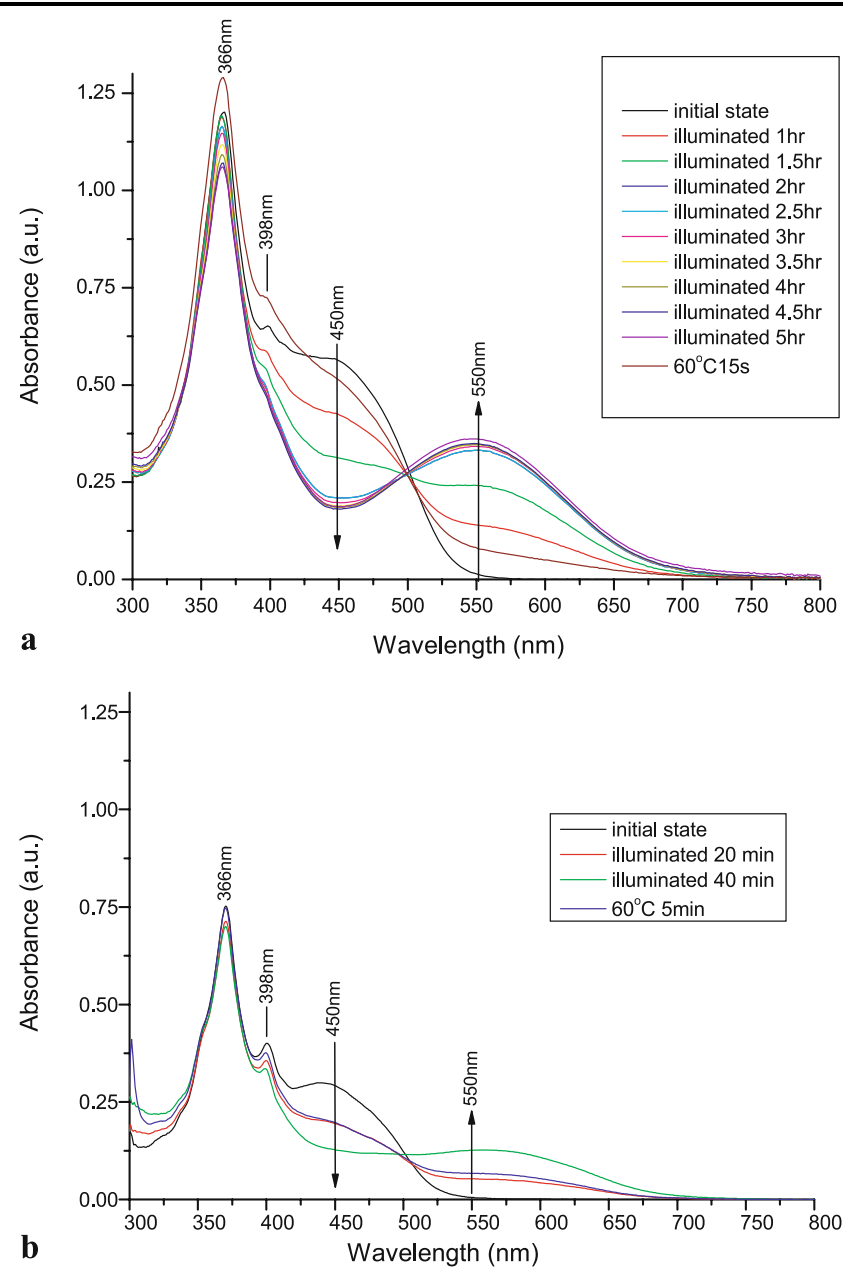

Fig. 3 (a) Optical absorption spectra of a dilute solution of 1 $\left(1.02 \times 10^{-5} \mathrm{~mol} / \mathrm{l}\right.$ in dichloromethane $)$ as a function of illumination time with a wavelength of $366 \mathrm{~nm}$ and after heating the illuminated solution for 15 seconds at $60^{\circ} \mathrm{C}$. (b) Optical absorption spectra of a saturated solution of $\mathbf{1}$ in 1, 2, 4-trichlorobenzene; illuminated at $450 \mathrm{~nm}$ wavelength; green curve for irradiated solution after thermal treatment for 5 minutes at $60^{\circ} \mathrm{C}$

(Fig. 4b). The bright features (corresponding to high tunneling probability) are attributed to the $\pi$-conjugated parts of the molecule [18], while the dark parts are attributed to the aliphatic side chains, which could not be resolved, since they are bent into the supernatant solution. However, for the solution irradiated at $450 \mathrm{~nm}$ for more than one hour, no change was observed on the molecular scale, which indicates that the cis-conformer cannot be incorporated thermodynamically stable into the monolayer at the interface, possibly due to the competition with the coexisting transconformers.

\section{Honeycomb lattices from polycyclic aromatic hydrocarbon derivatives}

In order to provide space for a conformational transition within a stable two-dimensional crystal on a solid surface, 
Fig. 4 STM images of a monolayer of $\mathbf{1}$ at the interface between an organic solution in 1, 2, 4-trichlorobenzene and the basal plane of HOPG. Sample bias $U_{s}=-1 \mathrm{~V}$; average tunneling current $I_{t}=0.1 \mathrm{nA}$. (a) Current image exhibiting characteristic double row pattern with grain boundaries reflecting the threefold-symmetry of the substrate. (b) High resolution height image revealing the nanophase separation between the flat lying hexa-peri-hexabenzocoronene cores on the one hand and the azobenzene moieties with their rigid linker on the other hand a

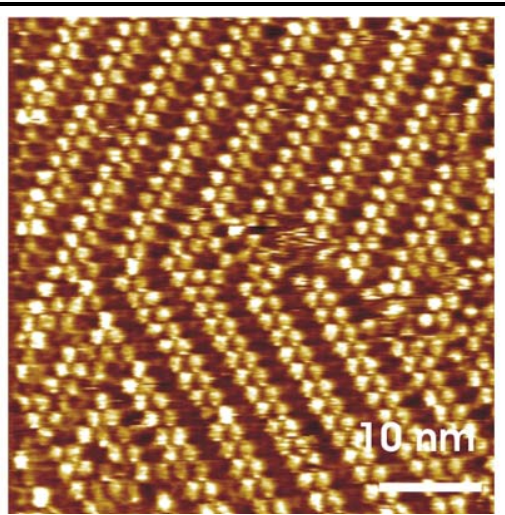

a robust molecular monolayer with nanometer-sized cavities is desirable, in which the conformational changes of azobenzene moieties can be achieved.

Porous molecular monolayers are used as two-dimensional hosts for molecular guests, which may be employed for molecular selection or the isolation of single molecules. They have been obtained from macrocycles at the interface between an organic solution and the basal plane of graphite, providing pores in the range of 1.2 to $2.7 \mathrm{~nm}$ [19-23]. Alternatively, supramolecular two-dimensional structures have been self-assembled at such solid-liquid interfaces, which are stabilized van der Waals interactions between pending alkyl chains [11, 24-28]. Thereby, cavities in the range of a few nanometers up to $5.4 \mathrm{~nm}$ have been obtained from identical molecular cores just by varying their side chains [27, 28]. However, due to the weakness of the interactions stabilizing the networks, the filling of the pores may be accompanied by structural transformations of the host [27]. More stable hosts were assembled from two molecular species interacting with each other by hydrogen bonding $[29,30]$ or metal coordination [31]; however, the size of the nanocavity cannot be tuned due to the specific molecule interactions.

We report here robust "honey-comb" structures at the interface between an organic solution and the basal plane of HOPG. The hosts are formed by $C_{3}$ symmetric polycyclic aromatic hydrocarbons (PAHs) stabilized by the weak interactions of alkyl side chains and thereby providing nanometer-sized cavities. Honey-comb networks from alkylated PAHs with ester end groups $\mathbf{2}$ have been filled with single coronenes as guest molecules without changing their lattice parameters, indicating both their robustness and their potential to isolate nanometer-sized single molecules.

The $C_{3}$ symmetric derivatives of diphenanthro[ $\left[3^{\prime}, 4^{\prime}, 5^{\prime}\right.$, $6^{\prime}$-efghi:3, 4, 5, 6-uvabc] ovalene with three zigzagperipheries ("trizigzagHBC") 3 have recently been shown to form self-assembled monolayers at the graphite-solution interface, exhibiting a "honey-comb" pattern with cavities on the nanometer scale [14]. In order to investigate the influence of the chemical periphery of trizigzagHBC on the pattern formation, compound $\mathbf{2}$ has been synthesized exhibiting ester end groups.

STM images obtained in-situ at the solid-liquid interface (Fig. 5a) reveal defect-free honeycomb-lattices of $\mathbf{3}$ on scales larger than $50 \times 50 \mathrm{~nm}^{2}$. The bright features (corresponding to high tunneling probability) are attributed to the $\pi$-conjugated aromatic rings [18], while the dark parts are attributed to the aliphatic side chains, which could not be resolved, probably since they partly bend into the supernatant solution, as well as due to their high conformational mobility on a time scale faster than the STM imaging. The image is characterized by hexagonal cavities, whose symmetry and orientation reflect the underlying HOPG lattice.

Molecules 2 possessing alkyl side chains with ester end groups similarly form very uniform $2 \mathrm{D}$ honey-comb monolayers (Fig. 5b). From the STM images the unit cell can be determined with high accuracy (Table 1). Most cavities appear empty, indicating that they are filled with solvent molecules exhibiting a large HOMO-LUMO gap and therefore contributing much less to the tunneling current than the PAH cores. However, some cavities appear filled by single molecules of $\mathbf{2}$, indicating that they may be also filled with different molecular guests.

For that purpose, coronene was added to the solution, because due to its relatively small HOMO-LUMO gap, it can potentially be recognized in the STM, while as a neat system it does not form a crystalline monolayer of flat lying molecules. Upon depositing a drop of the solution containing both 3 and $\mathbf{4}$, only the same structure as in the neat system $\mathbf{3}$ has been visualized, indicating no pore filling. However, $\mathbf{2}$ mixed with $\mathbf{4}$ provides a hexagonal lattice, which has the same lattice constant as that from the pure monolayer of 2 (Fig. 6, Table 1) but exhibits a different contrast with 
Fig. 5 STM current images of molecular monolayers self-assembled at the interface between organic solutions of (a) 3 and (b) 2 and the basal plane of HOPG. Both exhibit honey-comb structures consisting of two molecules in a unit cell as marked by parallelograms. In (b) most nanocavities appear empty (marked " $A$ "), while some are filled (marked " $B$ "). Sample bias $U_{s}=-1 \mathrm{~V}$; average tunneling current $I_{t}=0.1 \mathrm{nA}$
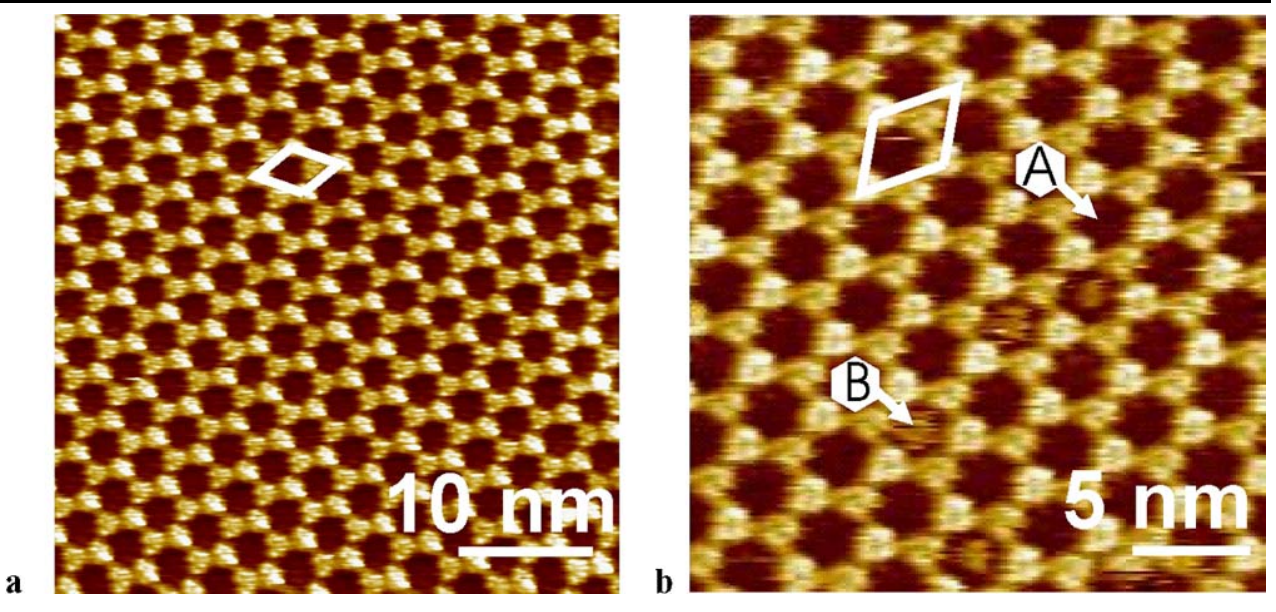

Fig. 6 STM current image at the interface between an organic solution and the basal plane of graphite, displaying the filling of the nanocavities of the honey-comb monolayer of 2 with single coronenes, 4. Sample bias $U_{s}=-1 \mathrm{~V}$; average tunneling current $I_{t}=0.1 \mathrm{nA}$

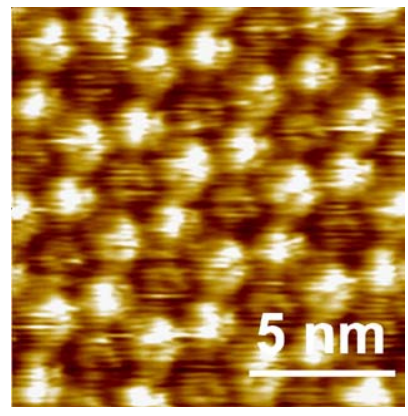

Table 1 Lattice constants of the two-dimensional crystal structures of the $\mathrm{HBC}$ derivatives

\begin{tabular}{lll}
\hline Compound & Lattice parameter & A (Area) in $\mathrm{nm}^{2}$ \\
\hline $\mathbf{2}$ & $\begin{array}{ll}a=3.31 \pm 0.14 \mathrm{~nm} \\
b=3.41 \pm 0.12 \mathrm{~nm}\end{array}$ & \\
& $\alpha=63 \pm 3^{\circ}$ & \\
& & \\
$\mathbf{3}$ & $a=3.34 \pm 0.14 \mathrm{~nm}$ & $10.25 \pm 0.60$ \\
& $b=3.49 \pm 0.16 \mathrm{~nm}$ & \\
& $\alpha^{\mathrm{a}}=62 \pm 2^{\circ}$ & \\
& & \\
$\mathbf{2}+\mathbf{4}$ & $a=3.34 \pm 0.07 \mathrm{~nm}$ & $9.92 \pm 0.24$ \\
& $b=3.44 \pm 0.02 \mathrm{~nm}$ & \\
& $\alpha=60 \pm 0.3^{\circ}$ & \\
\hline
\end{tabular}

${ }^{\mathrm{a}} \alpha$ : the angle between $a$ and $b$

The footprints of single $2, \mathbf{3}$, and 4 are $8.67 \mathrm{~nm}^{2}, 5.74 \mathrm{~nm}^{2}$, and $1.0 \mathrm{~nm}^{2}$, respectively

monodisperse, less bright objects in the nanocavities, instead of the dark and occasionally occupied voids within the monolayers of neat $\mathbf{2}$. Since the structure is only observed with the coronene $\mathbf{4}$, it is unlikely that the different contrast is only due to molecule $\mathbf{2}$ in two different states with regard to the $z$-axis, as was previously reported for other disc-like molecules [32]. It is rather concluded that the coronenes are entrapped in the molecular template provided by the unaltered honey-comb lattice of $\mathbf{2}$. In the STM image, the entrapped molecules are less bright than $\mathbf{2}$, and dark at their center. It has been reported previously that on HOPG, the center of coronene exhibits less tunneling current than the outer parts of the molecule [33], supporting further that single coronene molecules are entrapped within the cavities.

In Fig. 7, packing models are proposed, which are consistent with the experiment. The model for the packing of 3 (Fig. 7a) is constructed such that the conjugated PAH cores are oriented on HOPG as the nano-graphene layers in graphite. One of every two neighboring side chains is not drawn since there is not enough space for them on the surface. It appears that the size of the cavity in the network is not large enough to accommodate a coronene molecule. Thereby, in the mixture of $\mathbf{3}$ and $\mathbf{4}$, the pattern is the same as in the neat system 3. Figure $7 \mathrm{~b}$ displays the model for the $\mathbf{2}$ filled with $\mathbf{4}$. The conjugated core of $\mathbf{2}$ is packed as $\mathbf{3}$. However, only the part of the substituents until the fifth carbon from the PAH core are packed on HOPG, while the end groups of ester moieties have less affinity to HOPG and suspend in the solution, similarly to HBC with branched alkyl chains [34]. Therefore the formed cavity becomes larger to host a single coronene molecule. Consequently, the corresponding model $\mathrm{C}_{3}$ symmetric PAHs 2 containing ester end groups offer the possibility to host the guest molecules. Based on the above results, one or two substituted azobenzene moieties may be attached to a $\mathrm{C}_{3}$ symmetric PAH by esterization. The switchable moieties may then be hosted in the cavity with enough conformational freedom and will eventually influence the I-V characteristics through the conjugated $\mathrm{C}_{3}$ symmetric $\mathrm{PAH}$ cores. Thus a single molecular electronic device with switching characteristics may be obtained.

\section{Conclusions}

An HBC core linked rigidly to an azobenzene moiety is switchable in solution. The trans-conformer can be visu- 


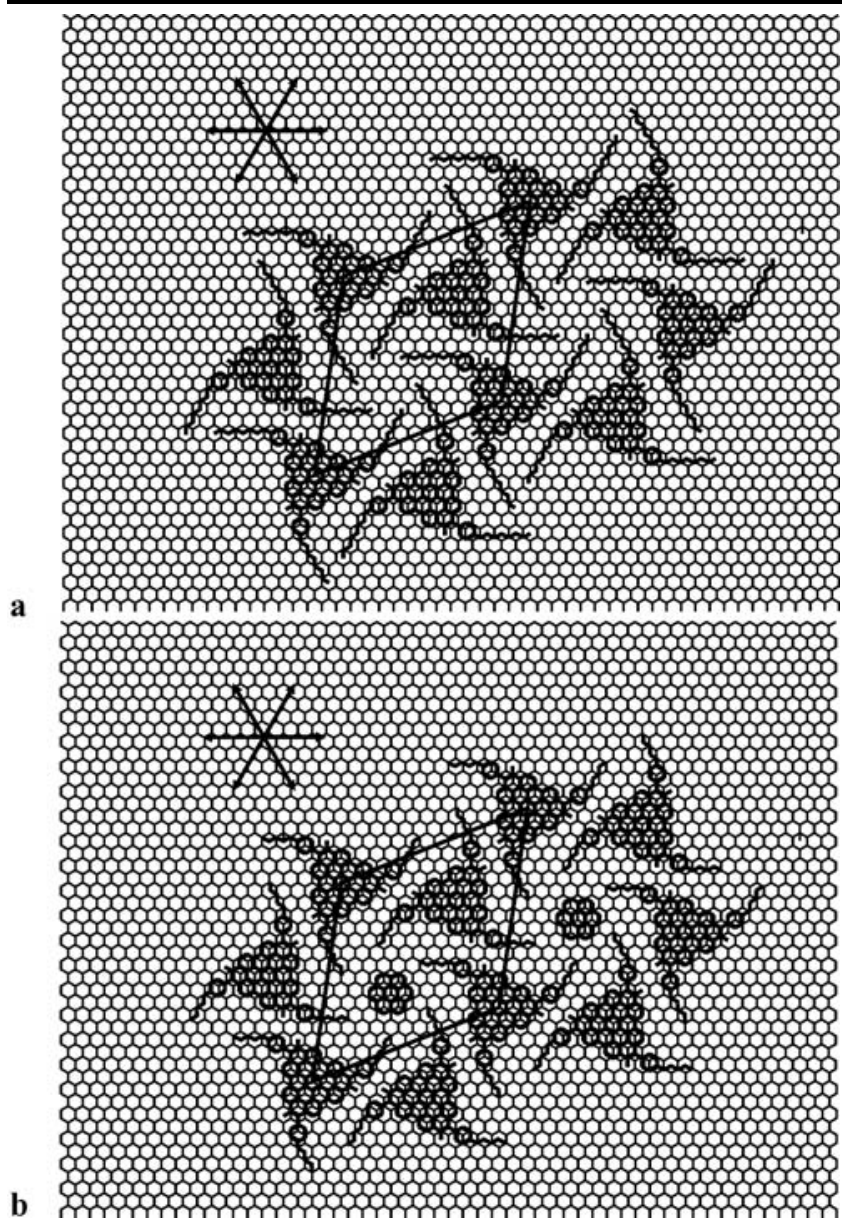

Fig. 7 Proposed molecular packing models for (a) neat 3 and (b) 2 coadsorbed with $4 . a=3.46 \mathrm{~nm}, b=3.46 \mathrm{~nm}, \alpha=60^{\circ}$

alized by STM at a solid-liquid interface, while the cisconformer cannot. This is attributed to constraints in the monolayer crystal of the trans-conformer. It may also be favored thermodynamically due to the perfectly flat conformation of the trans-conformer [35]. On the other hand, a stable and robust honey-comb architecture has been obtained. By functionalizing the end groups of its alkyl side chains we have controlled the capability of a honey-comb forming PAH to host a nanometer-sized guest molecule at the interface between an organic solution and HOPG. This method may be used to host single switchable moieties for applications in molecular electronics.

Acknowledgement Support by the Deutsche Forschungsgemeinschaft (SFB 658, SFB 625, Korean-German IRTG) is gratefully acknowledged.

Open Access This article is distributed under the terms of the Creative Commons Attribution Noncommercial License which permits any noncommercial use, distribution, and reproduction in any medium, provided the original author(s) and source are credited.

\section{References}

1. A. Nitzan, M.A. Ratner, Science 300, 1384 (2003)

2. R. Huber, M.T. Gonzale, S. Wu, M. Langer, S. Grunder, V. Horhoiu, M. Mayor, M.R. Bryce, C.S. Wang, R. Jitchati, C. Schönenberger, M. Calame, J. Am. Chem. Soc. 130, 1080 (2008)

3. M.A. Reed, C. Zhou, C.J. Muller, T.P. Burgin, J.M. Tour, Science 278, 652 (1997)

4. R.H.M. Smit, Y. Noat, C. Untiedt, N.D. Lang, M.C. van Hemert, J.M. van Ruitenbeek, Nature 419, 906 (2002)

5. A. Stabel, P. Herwig, K. Müllen, J.P. Rabe, Angew. Chem. Int. Ed. 34, 303 (1995)

6. A. Dhirani, P.-H. Lin, P. Guyot-Sionnest, R.W. Zehner, L.R. Sita, J. Chem. Phys. 106, 5249 (1997)

7. K. Müllen, J.P. Rabe, Acc. Chem. Res. 41, 511 (2008)

8. F. Jäckel, U.G.E. Perera, V. Iancu, K.-F. Braun, N. Koch, J.P. Rabe, S.-W. Hla, Phys. Rev. Lett. 100, 126102 (2008)

9. F. Jäckel, M.D. Watson, K. Müllen, J.P. Rabe, Phys. Rev. Lett. 92, $188303(2004)$

10. G.S. Kumar, D.C. Neckers, Chem. Rev. 89, 1915 (1989)

11. M. Alemani, M.V. Peters, S. Hecht, K.-H. Rieder, F. Moresco, L. Grill, J. Am. Chem. Soc. 128, 14446 (2006)

12. M.J. Comstock, N. Levy, A. Kirakosian, J. Cho, F. Lauterwasser, J.H. Harvey, D.A. Strubbe, J.M.J. Fréchet, D. Trauner, S.G. Louie, M.F. Crommie, Phys. Rev. Lett. 99, 038301 (2007)

13. G. Pace, V. Ferri, C. Grave, M. Elbing, C. von Hänisch, M. Zharnikov, M. Mayor, M.A. Rampi, P. Samorì, Proc. Natl. Acad. Sci. USA 104, 9937 (2007)

14. X. Feng, J. Wu, M. Ai, W. Pisula, L. Zhi, J.P. Rabe, K. Müllen, Angew. Chem. Int. Ed. 46, 3033 (2007)

15. J.P. Rabe, S. Buchholz, Science 253, 424 (1991)

16. Z. Wang, Ž. Tomović, M. Kastler, R. Pretsch, F. Negri, V. Enkelmann, K. Müllen, J. Am. Chem. Soc. 126, 7794 (2004)

17. X. Song, J. Perlstein, D.G. Whitten, J. Am. Chem. Soc. 119, 9144 (1997)

18. R. Lazzaroni, A. Calderone, J.L. Brédas, J.P. Rabe, J. Chem. Phys. 107, 99 (1997)

19. S. Höger, K. Bonrad, A. Mourran, U. Beginn, M. Möller, J. Am. Chem. Soc. 123, 5651 (2001)

20. E. Mena-Osteritz, P. Bäuerle, Adv. Mater. 13, 243 (2001)

21. P. Samori, F. Jäckel, O. Unsal, A. Godt, J.P. Rabe, Chem. Phys. Chem. 2, 461 (2001)

22. Ch. Grave, D. Lentz, A. Schäfer, P. Samorì, J.P. Rabe, P. Franke, A.D. Schlüter, J. Am. Chem. Soc. 125, 6907 (2003)

23. V. Kalsani, H. Ammon, F. Jäckel, J.P. Rabe, M. Schmittel, Chem. Eur. J. 10, 5481 (2004)

24. S. Ito, M. Wehmeier, J.D. Brand, Ch. Kübel, R. Epsch, J.P. Rabe, K. Müllen, Chem. Eur. J. 6, 4327 (2000)

25. F. Jäckel, M. Ai, J. Wu, K. Müllen, J.P. Rabe, J. Am. Chem. Soc. 127, 14580 (2005)

26. G. Schull, L. Douillard, C. Fiorini-Debuisschert, F. Charra, Nano Lett. 6, 1360 (2006)

27. S. Furukawa, K. Tahara, C. De Schryver, M. Van der Auweraer, Y. Tobe, S. De Feyter, Angew. Chem. Int. Ed. 46, 2831 (2007)

28. S. Lei, K. Tahara, F.C. De Schryver, K. Müllen, M. van der Auweraer, Y. Tobe, S. De Feyter, Angew. Chem. 47, 2964 (2008)

29. S.J.H. Griessl, M. Lackinger, F. Jamitzky, T. Markert, M. Hietschold, W.M. Heckl, Langmuir 20, 9403 (2004)

30. J.A. Theobald, N.S. Oxtoby, M.A. Phillips, N.R. Champness, P.H. Beton, Nature 424, 1029 (2003)

31. S. Stepanow, M. Lingenfelder, A. Dmitriev, H. Spillmann, E. Delvigne, N. Lin, X. Deng, C. Cai, J.V. Barth, K. Kern, Nat. Mater. 3, 229 (2004) 
32. P. Samorí, A. Fechtenkötter, F. Jäckel, T. Böhme, K. Müllen, J.P. Rabe, J. Am. Chem. Soc. 123, 11462 (2001)

33. K. Walzer, M. Sternberg, M. Hietschold, Surf. Sci. 415, 376 (1998)
34. F. Jäckel, PhD Dissertation, Humboldt University, Berlin (2005)

35. P. Samori, N. Severin, K. Müllen, J.P. Rabe, Adv. Mater. 12, 579 (2000) 